\title{
Tratamiento exitoso de una mucormicosis rinocerebral persistente en un paciente pediátrico durante el debut de una leucemia aguda
}

\author{
Fernanda Cofré, Milena Villarroel, Loreto Castellón y María E. Santolaya
}

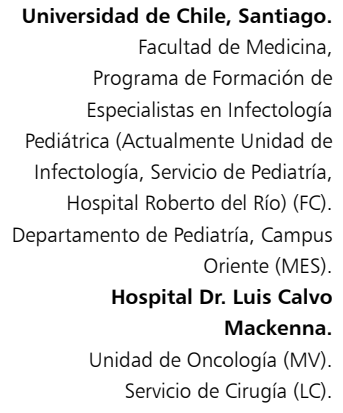

Universidad de Chile, Santiago.

Facultad de Medicina,

Programa de Formación de

Especialistas en Infectología Pediátrica (Actualmente Unidad de Infectología, Servicio de Pediatría,

Hospital Roberto del Río) (FC). Departamento de Pediatría, Campus Oriente (MES)

Hospital Dr. Luis Calvo Mackenna.

Unidad de Oncología (MV) Servicio de Cirugía (LC)

Financiamiento: sin financiamiento. Los autores declaran que no existen conflictos de interés.

Recibido: 2 de marzo de 2015 Aceptado: 4 de junio de 2015

Correspondencia a: Fernanda Cofré Segovia fcofre@yahoo.com

\section{Introducción}

$\mathrm{M}$ ucormicosis se refiere a la infección fúngica causada por hongos de la clase de los Zygomicetes, la que está compuesta por dos órdenes: Mucorales y Entomoftorales, ambos capaces de producir enfermedad en humanos ${ }^{1,2}$.

Desde la década de los 70 s, en acuerdo con los cambios taxonómicos, el término zigomicosis se ha usado como sinónimo de mucormicosis; sin embargo, actualmente se ha acordado utilizar el término mucormicosis o entomoftoramicosis para englobar aquellas infecciones causadas por mucorales $\mathrm{y} / \mathrm{o}$ entomoftorales que clínicamente pueden ser indistinguibles entre sí y no zygomicosis que hace referencia exclusiva a las infecciones por mucorales ${ }^{3}$.

Los mucorales causan mucormicosis, que se presenta habitualmente como una enfermedad fúngica invasora con rápida angio-invasión en pacientes inmunocomprometidos. Las especies de Mucorales que más frecuentemente producen enfermedad en humanos son Rhizopus spp. (dando cuenta de aproximadamente la mitad de los casos), Lichteimia (Absidia), Corymbifera y Mucor spp. ${ }^{1,2}$

Se presenta el caso de una niña preescolar que durante el debut de una leucemia linfoblástica aguda (LLA) evolucionó con una mucormicosis rinocerebral.

\section{Caso clínico}

Preescolar de sexo femenino, de 2 años, con diagnóstico de una LLA-L1 en tratamiento con prednisona y quimioterapia.

Con posterioridad a su primer ciclo de quimioterapia de inducción ingresó por un episodio de neutropenia febril de alto riesgo (NFAR), sin foco clínico evidente, destacando la presencia de una mucositis oral al examen físico. Se inició tratamiento antimicrobiano con ceftazidima, amikacina y cloxacilina. Se rescató un hemocultivo positivo para Staphylococcus warneri susceptible a oxacilina que se interpretó como contaminación. Evolucionó febril; a las $48 \mathrm{~h}$ de su ingreso presentó secreción conjuntival (con cultivo bacteriano y RPC para adenovirus negativos) a lo que se asoció un aumento de volumen palpebral derecho con movimientos oculares conservados y epistaxis en su fosa nasal derecha. Al cuarto día de evolución de su NFAR, por presentar una evolución desfavorable (persistencia de la fiebre y proteína C reactiva (PCR) en ascenso $(132 \mathrm{mg} / \mathrm{L})$ se cambió tratamiento antimicrobiano a vancomicina y piperacilina/ tazobactam, y se realizó estudio de enfermedad fúngica invasora (EFI). La tomografía axial computada (TAC) de cavidades paranasales reveló una sinusitis maxilar y etmoidal derecha sin compromiso orbitario (Figura 1). La TAC de tórax, ecografía abdominal y fondo de ojo 
fueron normales. Las dos muestras de galactomanano (GM) en días alternos resultaron negativas.

Al séptimo día de evolución de su NFAR se constató una lesión ulcerada necrótica no dolorosa en el paladar duro (Figura 2), de la que se realizó biopsia junto con efectuar biopsia de la mucosa nasal derecha. Frente a la sospecha de EFI se comenzó una disminución progresiva de prednisona y se solicitó al equipo de otorrinolaringología realizar un drenaje amplio de los senos paranasales. Se inició tratamiento antifúngico con voriconazol iv y anfotericina $\mathrm{B}$ deoxicolato $(\mathrm{AmB}) 0,5 \mathrm{mg} / \mathrm{kg} /$ día, por no contar en ese momento con la formulación lipídica de AmB. El estudio histológico de la biopsia mostró hifas cenocíticas, gruesas, con ángulos en $90^{\circ}$ en la muestra de la mucosa perioral y el hueso maxilar, con cultivo negativo. Con el diagnóstico histopatológico de mucormicosis se ajustó la terapia antifúngica: se suspendió voriconazol y se inició AmB en dispersión coloidal, a dosis de $5 \mathrm{mg} /$ $\mathrm{kg}$ /día. La resonancia magnética $(\mathrm{RM})$ cerebral descartó compromiso de SNC en ese momento.

Se realizó por vía endoscópica una maxilectomía parcial medial con resección del cornete inferior y medio y de la pared medial del seno maxilar derecho. La descripción macroscópica fue "compromiso de la lámina papirácea y hueso decolorado subyacente a la lesión del paladar”. El cultivo fue positivo para Rhizopus arrhizus (ex oryzae) (Figura 3 a y 3 b). Desde ese momento se cambió AmB dispersión coloidal a AmB liposomal, en igual dosis; por el compromiso óseo se agregó posaconazol $(20 \mathrm{mg} / \mathrm{kg} /$ día) y se programó una cirugía de resección amplia que incluyó maxilectomía derecha ampliada con resección del laberinto etmoidal derecho y traqueotomía (Figura 4). La biopsia mostró elementos fúngicos y el cultivo fue positivo para Rhizopus arrhizus. Una semana más tarde se sometió a una nueva cirugía en que se realizó un aseo quirúrgico con resección del tejido necrótico y reparación de una fístula de LCR que se hizo evidente durante el procedimiento; la biopsia etmoidal fue positiva por histología y cultivo. Por sospecha de compromiso de SNC se aumentó la dosis de AmB liposomal a $10 \mathrm{mg} / \mathrm{kg} /$ día. Posteriormente a ello se mantuvo con tratamiento antifúngico asociado y con aseos quirúrgicos y cambio de packing de la zona operatoria semanal.

A los 45 días de evolución presentó clínicamente un aumento de volumen palpebral derecho. Se realizó una TAC de cerebro que mostró compromiso de la tabla externa del hueso frontal. Se sometió a un nuevo aseo quirúrgico y se realizó resección parcial del hueso frontal y curetaje; la biopsia rápida nuevamente resultó positiva al mismo hongo.

Se consultó el caso con expertos internacionales y considerando la persistencia de la infección con muestras histológicas y microbiológicas positivas luego de 45 días de tratamiento antifúngico se decidió asociar caspofungi-

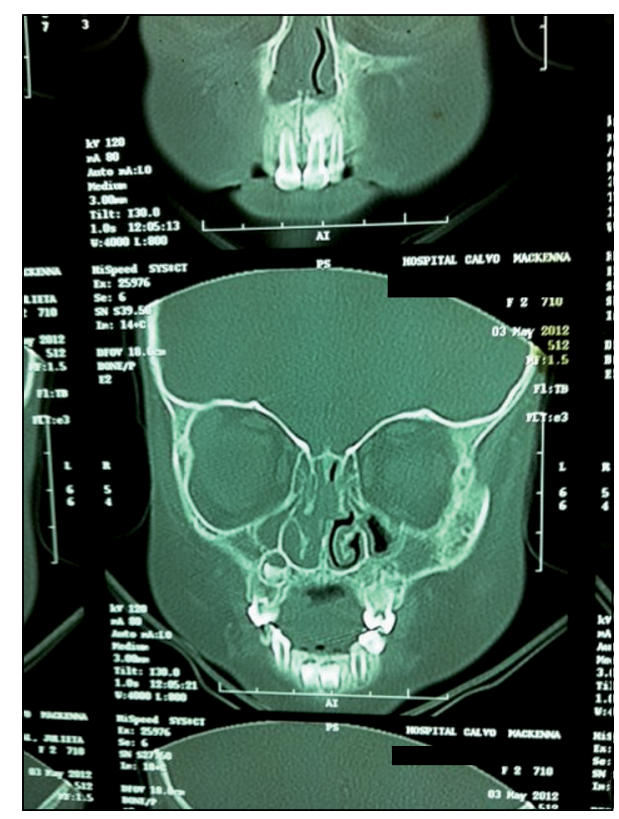

Figura 1. Tomografía axial computada de cavidades paranasales con sinusitis maxilar y etmoidal derecha sin compromiso orbitario.

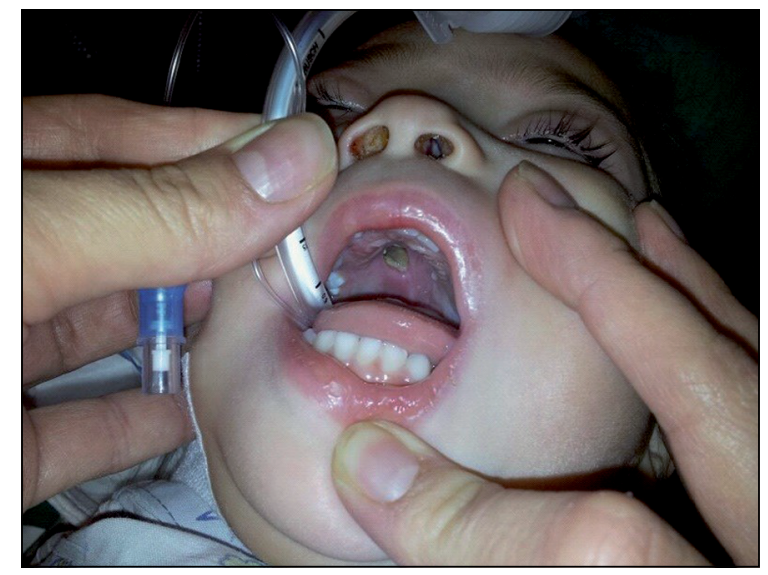

Figura 2. Lesión ulcerada, necrótica, no dolorosa en el paladar duro es característica de mucormicosis.
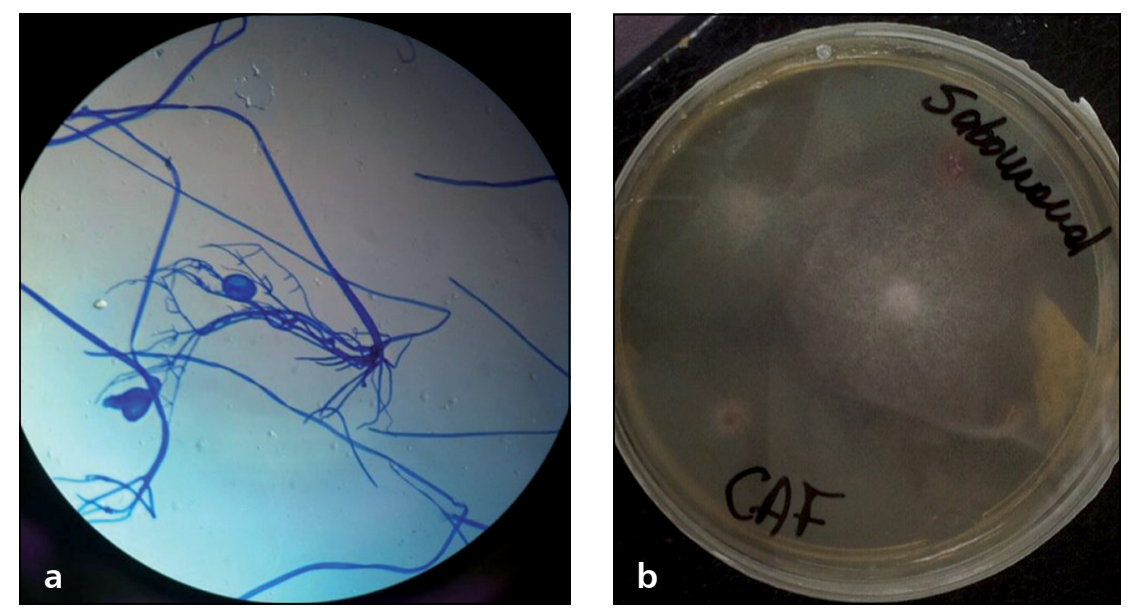

Figura 3a y 3b. Microscopia (a) y cultivo (b) positivos para Rhizopus arrhizus. 


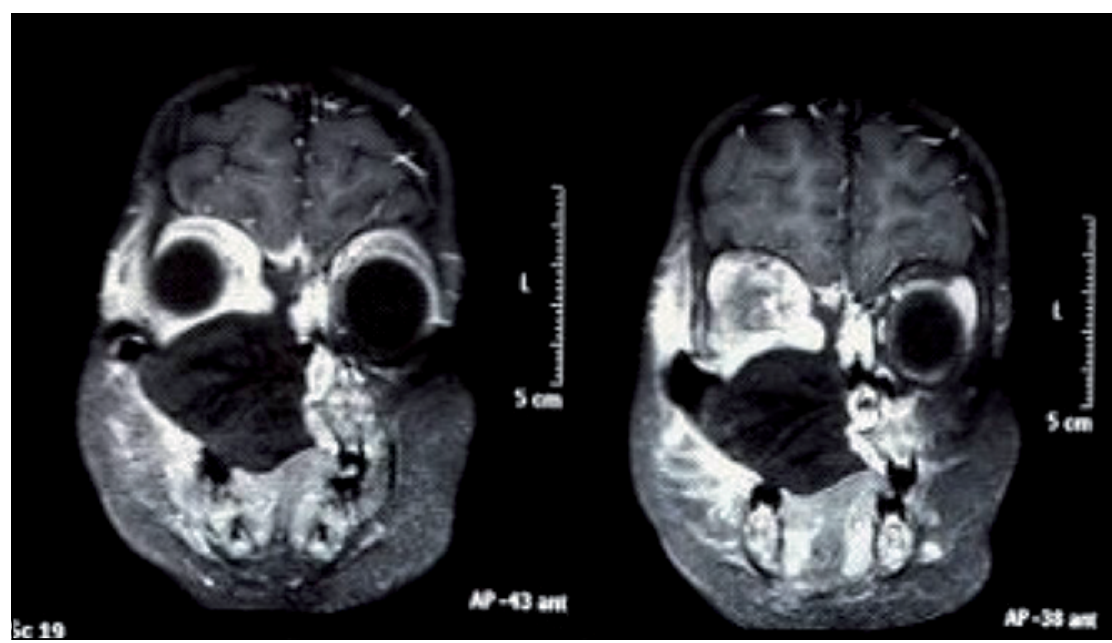

Figura 4. Cirugía amplia de resección maxilar.

Tabla 1. Factores de riesgo relacionados a las diferentes formas clínicas de presentación de mucormicosis

\begin{tabular}{|ll|}
\hline Factores predisponentes & Localización y forma clínica \\
Cetoacidosis diabética & Rinocerebral \\
Neutropenia & Pulmonar y diseminada \\
Corticoesteroides & Pulmonar, rinocerebral y diseminada \\
Deferoxamina & Diseminada \\
Malnutrición & Gastrointestinal \\
Traumatismos, catéteres, sitios de inyección y piel macerada & Cutánea y subcutánea \\
\hline
\end{tabular}

na $50 \mathrm{mg} / \mathrm{m}^{2} /$ día a AmB liposomal. Se mantuvo con aseos quirúrgicos semanales.

La paciente evolucionó clínicamente bien, con controles de imágenes sin cambios y sin evidencia de recaída microbiológica. Al día 106 de terapia se efectuó la reconstrucción del maxilar y piso de la órbita del lado derecho, con muestras histológicas y microbiológicas negativas. Se suspendió caspofungina y se mantuvo con AmB liposomal diaria. A los 148 días reinició ciclos de quimioterapia (mielograma sin blastos) y tras 12 procedimientos quirúrgicos y 170 días de tratamiento antifúngico se suspendió AmB y se consideró tratada. Se acordó usar profilaxis secundaria durante los períodos de neutropenia asociados a quimioterapia con posaconazol oral (18 mg/ $\mathrm{kg}$ /día monitorizando su concentración plasmática) en forma ambulatoria o con AmB liposomal en los episodios de neutropenia en que necesitó estar internada.

\section{Discusión}

La mucormicosis es una de las enfermedades fúngicas invasoras menos prevalentes (8-13\% en autopsias) pero con las mayores tasas de mortalidad (56-64\% en pediatría $)^{4,5}$

En niños, la mucormicosis es una enfermedad inusual y la mayoría de los reportes son casos aislados. Se presenta en escenarios similares a los adultos: enfermedades hematológicas malignas, trasplante de precursores hematopoyéticos, diabetes mellitus descompensada, sobrecarga de fierro, trauma, uso prolongado de corticosteroides, uso de drogas intravenosas, malnutrición, recién nacidos de alto riesgo y portadores de aciduria metabólica congénita ${ }^{6,7}$. Actualmente las enfermedades hematológicas dan cuenta de $60 \%$ de los casos como principal factor predisponente ${ }^{8}$. Son factores de riesgo independientes de mortalidad en pediatría el ser lactantes bajo el año de edad y la infección diseminada $a^{4,7-9}$.

La emergencia y reconocimiento creciente de la mucormicosis en los últimos 5 a 10 años en pacientes inmunocomprometidos están asociados a una mayor sospecha diagnóstica y al creciente uso de voriconazol y equinocandinas en forma profiláctica en este grupo de pacientes, lo que podría explicar su aumento relati$\mathrm{vo}^{4,5,7,10,11}$. Se ha demostrado que voriconazol es capaz de inducir la expresión de un fenotipo hipervirulento en los Zigomycetes evidenciado por una mayor mortalidad y compromiso pulmonar en moscas de la fruta y ratones, lo que no ocurre al exponerlos a otros antifúngicos como itraconazol, caspofungina y $\mathrm{AmB}^{11,12}$.

La infección generalmente se produce por la inhalación de las esporangioesporas desde el aire o por inoculación directa en la piel o mucosa dañada ${ }^{5}$. Según la presentación clínica y el sitio anatómico comprometido, existen seis formas de presentación: rinocerebral, pulmonar, cutánea, gastrointestinal, diseminada y miscelánea o atípica ${ }^{5,10}$ y cada presentación se asocia a factores de riesgo específicos $(\text { Tabla } 1)^{4,10}$.

\section{Mucormicosis rino-cerebral}

La mucormicosis rino-cerebral es la presentación clínica más frecuente. Generalmente es de comienzo agudo, con un cuadro clínico compatible con una sinusitis aguda o una celulitis periorbitaria que se acompaña de dolor facial u ocular, secreción conjuntival, visión borrosa y edema de tejidos blandos ${ }^{3,10,13,14}$. Los senos más frecuentemente comprometidos son los etmoidales y maxilares ${ }^{15}$.

Sin tratamiento la infección se disemina desde la lámina etmoidal hacia la órbita causando pérdida de la movilidad de la musculatura extraocular y proptosis asociada a quemosis. La infección también se puede diseminar desde el seno paranasal a la cavidad oral a través del paladar duro generando una úlcera necrótica y dolorosa como ocurrió en nuestro paciente ${ }^{3,10}$ y en forma retrógrada hacia el sistema nervioso central (SNC) provocando trombosis del seno cavernoso, compromiso de la arteria carótida interna con trombosis y extensos infartos 
cerebrales $^{3,10}$. El compromiso orbitario contralateral y de los pares craneanos es un signo ominoso que sugiere una trombosis del seno cavernoso ${ }^{10}$.

El daño y diseminación de la infección se producen porque el hongo es capaz de penetrar las paredes arteriales, separando la íntima de la media y generando trombosis, infartos y necrosis ${ }^{13}$.

En $85 \%$ de los casos se asocia a diabetes mellitus descompensada o a cetoacidosis diabética y también se da en el contexto de pacientes con neutropenia. Tiene una mortalidad hasta de $85 \%$, siendo $R$. arrhizus la especie prevalente $^{14}$.

El diagnóstico de zigomicosis invasora es especialmente difícil ya que requiere exámenes histopatológicos que no suelen estar disponibles de inmediato ${ }^{15}$. El diagnóstico definitivo se hace por la identificación morfológica directa del hongo y su recuperación desde un cultivo obtenido desde el sitio presuntamente comprometido ${ }^{3}$. La falta de sospecha clínica y la limitación de las técnicas diagnósticas explican que hasta en $50 \%$ de los casos el diagnóstico sea post mortem $^{15,16}$.

La evaluación con imágenes es necesaria para definir la extensión anatómica de la infección. La TAC o RM son lo mejor para definir la extensión y guiar el desbridamiento quirúrgico ${ }^{3}$.

No existen ensayos clínicos aleatorios en adultos o niños que guíen las decisiones terapéuticas. Los principios generales para un tratamiento exitoso son: el diagnóstico precoz, la reversión de los factores predisponentes, el desbridamiento quirúrgico amplio y el tratamiento antifúngico precoz $z^{3,6,14,17,18}$.

Las formulaciones lipídicas de AmB se encuentran actualmente licenciadas para el tratamiento de los pacientes con EFI y como tratamiento empírico para pacientes con neutropenia persistente ${ }^{19}$. Las indicaciones para el uso de AmB liposomal como primera línea terapéutica son la aspergilosis invasora, la candidiasis invasora y la mucormicosis. Las formulaciones lipídicas de AmB se usan como tratamiento primario en las mucormicosis invasoras y aunque la dosis óptima es desconocida, se sugiere utilizar dosis de $5 \mathrm{a} 10 \mathrm{mg} / \mathrm{kg}$ de AmB liposomal y 5 a $7 \mathrm{mg} / \mathrm{kg} / \mathrm{dia}$ de AmB complejo lipídico $3,7,17,19$. En la literatura especializada no se especifican plazos de tratamiento y más bien se enfatiza la necesidad de tratar a los pacientes hasta controlar adecuadamente los factores que favorecieron la aparición de la infección, junto con evidenciar mejoría clínica y microbiológica, además de resolución de las imágenes.

La mayoría de los azoles, incluyendo fluconazol y voriconazol, no tienen actividad significativa contra los Zygomycetes. Posaconazol, un azol de amplio espectro y disponible vía oral, parece tener una potente actividad contra este género de hongos y puede ser usado como una alternativa para el tratamiento de la mucormicosis; es importante considerar que su uso en niños bajo los 13 años aún está dentro de la categoría off label por la FDA ${ }^{3}$. Un estudio multicéntrico pediátrico logró mostrar tasas de éxito de 60 a $70 \%$ con posaconazol como terapia de rescate para la zigomicosis en pacientes con EFI probada o probable que previamente habían recibido por lo menos dos antifúngicos en forma combinada o secuencial ${ }^{7,17,20}$. Se propone que el uso de posaconazol permitiría reducir la dosis de AmB liposomal generando una mejor tolerancia $\mathrm{y}$ adherencia al tratamiento ${ }^{17}$.

Actualmente se encuentra en desarrollo (estudio fase 3) un nuevo antifúngico de la familia de los azoles. Isavuconazol (BAL 4851) es el compuesto activo del compuesto isavuconazonium sulfato, que es convertido en el plasma a isovuconazol por acción de las esterasas y actúa sobre la citocromo P450 fúngica ${ }^{19}$. Tiene un espectro antifúngico amplio (Candida spp, Aspergillus spp, Cryptococcus spp y Mucorales) in vivo e in vitro por lo que se propone como tratamiento de las EFI, incluyendo la aspergilosis invasora y la mucormicosis, especialmente en aquellos pacientes que no son capaces de tolerar el tratamiento con AmB o en forma combina$\mathrm{da}^{19-21}$. Su actividad antifúngica es comparable a AmB en modelos murinos de mucormicosis, específicamente para $R$. delamar ${ }^{20}$. Se encuentra disponible para administración intravenosa $\mathrm{u}$ oral, tiene farmacocinética linear y vida media prolongada lo que permite su administración una vez por día. Puede ser administrado en pacientes con insuficiencia renal ${ }^{19,21}$.

Las equinocandinas no tienen actividad in vitro contra los mucorales; sin embargo, los estudios tradicionales de susceptibilidad podrían no reflejar la verdadera actividad de las equinocandinas contra los hongos filamentosos en general y contra los agentes productores de mucormicosis en particular. Existen reportes de que $R$. oryzae, el principal agente etiológico de mucormicosis, es capaz de expresar el blanco de acción de las equinocandinas; la 1,3 $\beta$-D glucano sintetasa; codificado por el gen FKS y que caspofungina inhibiría la actividad de la enzima in vitro $\mathrm{y}$ tendría actividad in vivo en modelos murinos diabéticos de mucormicosis diseminada $a^{22,23}$.

Existen dos posibles mecanismos que explicarían por qué la adición de una equinocandina podría mejorar la eficacia del tratamiento con $\mathrm{AmB}$ en la mucormicosis: por disrupción de los enlaces $\beta$-glucano de la pared celular permitiendo una mayor llegada de AmB a la membrana celular, y por alteración de la virulencia del hongo retrasando la filamentación o alterando el contenido de la pared celular ${ }^{22}$.

La asociación de caspofungina con AmB en complejo lipídico ha mostrado sinergia in vitro y eficacia en los mismos modelos murinos ${ }^{22,23}$. En adultos se ha podido comprobar que pacientes tratados con la combinación polieno-caspofungina tienen una mejor sobrevida a los 30 
días del alta y a largo plazo. No está claro si este efecto es sólo de caspofungina o común a todo el grupo de las equinocandinas ${ }^{22}$.

\section{Comentario}

En nuestra paciente la persistencia de compromiso de senos paranasales y SNC con estudios histológico, microbiológico o imagenológico positivos pese a un tratamiento prolongado y en dosis adecuadas $(10 \mathrm{mg} / \mathrm{kg} /$ día $)$ llevó a la decisión de realizar un tratamiento de rescate combinado que finalmente resultó ser exitoso desde el punto de vista clínico y microbiológico.

No podemos afirmar causalidad entre la mejoría clínica y microbiológica de la paciente y el uso de terapia combinada con AmB liposomal y caspofungina; sólo podemos describir que pese a suspender la quimioterapia, disminuir la dosis de corticosteroides, realizar cirugías radicales y aseos quirúrgicos repetidos, unido a terapia combinada con AmB liposomal más posaconazol, la paciente permaneció con muestras histológicas y microbiológicas positivas luego de 146 días de terapia. Con posterioridad se asistió a una mejoría clínica y microbiológica coincidentes con el uso de terapia combinada de AmB liposomal más caspofungina, la que se prolongó por 52 días. A la fecha de este manuscrito, la paciente se encuentra nuevamente con quimioterapia con diagnóstico de recaída de su LLA, ha mantenido su profilaxis secundaria en períodos de neutropenia con AmB liposomal o posaconazol y no ha presentado evidencias de reactivación de su enfermedad fúngica.

\section{Resumen}

Los hongos del orden Mucorales causan la mucormicosis, que se presenta habitualmente como una enfermedad fúngica invasora con rápida angioinvasión en pacientes inmunocomprometidos. La presentación rino-cerebral es la más frecuente. Las formulaciones lipídicas de anfotericina B se usan como tratamiento primario en las mucormicosis invasoras; el uso combinado de posaconazol podría permitir reducir la dosis de anfotericina $\mathrm{B}$ generando una mejor tolerancia y adherencia al tratamiento. La asociación de caspofungina con anfotericina ha demostrado acción sinérgica in vitro y eficacia en modelos murinos.

Se presenta el caso de una niña preescolar que durante el debut de una leucemia linfoblástica aguda evolucionó con una mucormicosis rino-cerebral persistente, que respondió en forma exitosa al tratamiento antifúngico combinado de anfotericina liposomal y caspofungina.

\section{Referencias bibliográficas}

1.- Naggie S, Perfect J R. Molds: hyalohyphomycosis, phaeohyphomycosis, and zygomycosis. Clin Chest Med 2009; 30: 337-53.

2.- González C E, Rinaldi M G, Sugar A M. Zygomycosis. Infect Dis Clin North Am 2002; 16: 895-914.

3.- Kwon-Chung K J. Taxonomy of fungi causing mucormycosis and entomophthoramycosis (zygomycosis) and nomenclature of the disease: molecular mycologic perspectives. Clin Infect Dis 2012; 54 Suppl 1: S8-15.

4.- Däbritz J, Attarbaschi A, Tintelnot K, Kollmar N, Kremens B, von Loewenich F D, et al. Mucormycosis in paediatric patients: demographics, risk factors and outcome of 12 contemporary cases. Mycoses 2011; 54: e785-8.

5.- Petrikkos G, Skiada A, Lortholary O, Roilides E, Walsh T J, Kontoyiannis D P. Epidemiology and clinical manifestations of mucormycosis. Clin Infect Dis 2012; 54 Suppl 1: S23-34.

6.- Katragkou A, Roilides E. Best practice in treating infants and children with proven, probable or suspected invasive fungal infections. Curr Opin Infect Dis 2011; 24: 225-9.

7.- Zaoutis T E, Roilides E, Chiou C C, Buchanan W L, Knudsen T A, Sarkisova T A, et al. Zygomycosis in children: a systematic review and analysis of reported cases. Pediatr Infect Dis J 2007; 26: 723-7.

8.- Klimko N N, Khostelidi S N, Volkova A G, Popova M O, Bogomolova T S, Zuborovskaya L S, et al. Mucormycosis in haematological patients: case report and results of prospective study in Saint Petersburg, Russia. Mycoses 2014; 57 Suppl 3: 91-6.

9.- Bonifaz A, Tirado-Sánchez A, Calderón L, Romero-Cabello R, Kassack J, Ponce R M, et al. Mucormycosis in children: a study of 22 cases in a Mexican hospital. Mycoses 2014; 57 Suppl 3: 79-84.

10.- Kontoyiannis D P, Lionakis M S, Lewis R E, Chamilos G, Healy M, Perego C, et al. Zygomycosis in a tertiary-care cancer center in the era of Aspergillus-active antifungal therapy: a case-control observational study of 27 recent cases. J Infect Dis 2005; 191: 1350-60.

11.- Spellberg B, Edwards J Jr, Ibrahim A. Novel perspectives on mucormycosis: pathophysiology, presentation and management. Clin Microbiol Rev 2005; 18 : 556-69.

12.- Perfect J R. The impact of the host on fungal infections. Am J Med 2012; 125 Suppl 1: S39-51.

13.- Prasad P A, Vaughan A M, Zaoutis T E. Trends in zygomycosis in children. Mycoses 2012; 55: $352-6$

14.- Ibrahim M, Chitnis S, Fallon K, Roberts T. Rhinocerebral mucormycosis in a 12 -year-old girl. Arch Neurol 2009; 66: 272-3.

15.- Bonifaz A. Capítulo 28: Mucormicosis y Entomoftoromicosis (Zigomicosis) Micología Médica Básica, 4a Edición, Mc Graw-Hill, México; 2012: pág 399-426.

16.- Torres-Narbona M, Guinea J, Muñoz P, Bouza E. Zigomicetos y zigomicosis en la era de las nuevas terapias antifúngicas. Rev Esp Quimioter 2007; 20: 375-86.

17.- Georgala A, Vekemans M, Husson M, Meuleman N, Beguin H, Nolard N, et al . Zygomycosis in the immunocompromised patient: a case report. Acta Biomed 2006; 77 : Suppl 2: 5-9.

18.- Thomas S, Singh V D, Vaithilingam Y, Thayil S C, Kothari R. Rhinocerebral mucormycosis-a case report. Oral Maxillofac Surg 2012; 16: 233-6. 
19.- Fortún J, Carratalá J, Gavaldác J, Lizasoain M, Salavert M, de la Cámara R, et al.

Recomendaciones sobre el tratamiento de la enfermedad fúngica invasiva por Aspergillus spp. y otros hongos filamentosos de la Sociedad Española de Enfermedades Infecciosas y Microbiología Clínica (SEIMC). Actualización 2011. Enferm Infecc Microbiol Clin 2011; 29: 435-54.

20.- Anaissie E, Mc Ginnis M, Pfaller M. Zygomycosis. En: Clinical Mycology. Second Edition. Pág 297-307.

21.- Lehrnbecher T, Attarbaschi A, Duerken M, Garbino J, Gruhn B, Kontny U, et al.
Posaconazole salvage treatment in paediatric patients: a multicentre surrey. Eur J Clin Microbiol Infect Dis 2010; 29: 1043-5.

22.- Peixoto D, Gagne L S, Hammond S P, Gilmore E T, Joyce A C, Soiffer R J, et al. Isavuconazole treatment of a patient with disseminated mucormycosis. J Clin Microbiol 2014; 52: 1016-9.

23.- Luo G, Gebremariam T, Lee H, Edwards J E $\mathrm{Jr}$, Kovanda L, Ibrahim A S. Isavuconazole therapy protects immunosuppressed mice from mucormycosis. Antimicrob Agents Chemother 2014; 58: 2450-3.

24.- Falci D R, Pasqualotto A C. Profile of isavuconazole and its potential in the treatment of severe invasive fungal infections. Infect Drug Resist 2013; 6: 163-74.

25.- Reed C, Bryant R, Ibrahim A S, Edwards J Jr., Filler S G, Goldberg R, et al. Combination polyene-caspofungin treatment of rhino-orbitalcerebral mucormycosis. Clin Infect Dis 2008; 47: 364-71.

26.- Spellberg B, Fu Y, Edwards J E Jr, Ibrahim A S. Combination therapy with amphotericin B lipid complex and caspofungin acetate of disseminated zygomycosis in diabetic ketoacidotic mice. Antimicrob Agents Chemother 2005: 49: 830-2. 